\title{
Satisfacción del cliente de la consulta externa de pediatría en clínicas privadas
}

\author{
Morillo Castro, Jorge Luis* \\ Marcano Aular, Yelitza**
}

\section{Resumen}

El presente estudio tiene como objetivo determinar el nivel de satisfacción del cliente de las consultas pediátricas que funcionan en las clínicas privadas del Municipio Carirubana del Estado Falcón. Se trata de una investigación transversal, no experimental, cuantitativa, descriptiva, con diseño de campo. La población correspondió a las personas que solicitaron el servicio en las consultas externas de Pediatría que funcionan en las clínicas: Policlínica de Especialidades, Clínica Falcón y Clínica La Familia. La muestra fue de carácter intencional conformada por 64 clientes. Fue diseñado y aplicado un cuestionario de 44 ítems, a las unidades informantes o acompañantes de los pacientes que recibieron el servicio médico. Según los resultados obtenidos, el perfil sociodemográfico de los clientes corresponde a: madre entre 18 a 30 años de edad, con estudios de educación superior, que acude a la consulta más de dos veces al año, para el control de niño sano, proceden del medio urbano, se moviliza en vehículo propio o público hasta la clínica, no es referida por empresa o seguro y selecciona la consulta por recomendación de familiares o amigos. El rendimiento percibido por la mayoría de los clientes fue positivo, se cumplieron sus expectativas, expresando satisfacción por la mayoría de los aspectos del servicio y mostrando algunos rasgos de fidelidad.

Palabras clave: Satisfacción del cliente, expectativas, rendimiento percibido, fidelidad de servicio.

\section{Recibido: 12-07-10. Aceptado: 02-12-10}

Médico Especialista en Pediatra y Puericultura. MSc. en Gerencia de Empresas. e-mail: jorge morillo@yahoo.es.

** Ingeniero de Sistemas. Especialista en Costos. MSc. en Gerencia de Empresas. Doctorante en Informática. Profesora Asociada de la Universidad del Zulia. Núcleo Punto Fijo. e-mail: ymarcanoa@hotmail.com.. 


\section{Client Satisfaction with Pediatric Outpatient Consults at Private Clinics}

\section{Abstract}

The objective of this study is to determine the level of client satisfaction with pediatric consults functioning at private clinics in the Carirubana Municipality in the State of Falcón. The research was transversal, non-experimental, quantitative and descriptive with a field design. The population corresponded to people who requested service at the pediatric outpatient consults functioning in the following clinics: the Polyclinic for Specializations, Falcon Clinic and the Family Clinic. The sample was intentional, composed of 64 clients. A questionnaire with 44 items was designed and applied to the informing units or patient companions who received the medical service. According to the results obtained, the client socio-demographic profile corresponded to: a mother between 18 and 30 years of age with higher education studies, who recurs to consults more than twice a year to monitor the healthy growth of a child, who comes from an urban environment, travels to the clinic in her own or in a public vehicle, who is not referred to the consult by a company or insurance plan and who chooses the consulting services through the recommendation of family or friends. A majority of patients perceived the clinic's performance as positive, fulfilling expectations. They expressed satisfaction with most aspects of the services and demonstrated some traits of loyalty.

Key words: Client satisfaction, expectations, perceived performance, service loyalty.

\section{Introducción}

Para cualquier tipo de organización, la satisfacción es un requisito indispensable para ganarse un lugar en la mente de los clientes y por ende, en el mercado meta (Labrador, 2006) y viene a constituir el nivel del estado de ánimo que resulta de comparar el rendimiento percibido de servicio con sus experiencias (Kotler, 1993; Huete, 2001). Un cliente satisfecho trae consigo la lealtad, la difusión gratuita comunicando a otros, sus experiencias positivas con el servicio. Además, genera en él una determinada participación en el mercado, siendo que el cliente satisfecho deja de lado a la competencia (Thompson, 2005).

Es así como, el término satisfacción del cliente engloba el rendimiento percibido, las expectativas y los niveles de satisfacción, dada la satisfacción por el grado en que se cubran expectativas previas del cliente, mientras que el rendimiento percibido se considera implícito o como mediador entre expectativas y satisfacción (Oliver, 1980; Peralta, 2006).

Por otro lado, es necesario referir que el servicio es catalogado como "todo acto o función que una parte puede ofrecer a otra, que es esencialmente intangible y no da como resultado ninguna propiedad. Su producción puede o no vincularse a un producto" (Kotler, 1993; 511). Cabe destacar que en el área de salud, el servicio de atención es intangible, el cliente es el único juez de su calidad y debe contar con profesionales de un alto nivel de excelencia, un eficiente uso de los recursos humanos, un mínimo de riesgo para el paciente, un alto grado de satisfacción por parte del usuario y un 
impacto final en salud (Horovitz y Jurgens, 1999; World Health Organization, 2006).

En este contexto, tomando en cuenta el análisis de los documentos seleccionados y los conocimientos y experiencias de los autores es posible plantear las siguientes situaciones de causaefecto en el sector salud privado: Desde hace algunos años, las clínicas del país han tenido dificultades en su funcionamiento, dado el aumento exacerbado en la demanda de atención de hasta un $80 \%$, llegando incluso a sobrepasar el $95 \%$ de su capacidad de atención. Esta situación de saturación y casi colapso se ha atribuido principalmente a la insatisfacción de los usuarios por el servicio brindado en los centros asistenciales de carácter público, principalmente en la atención curativa, ya que en las consultas preventivas no es tan marcada esta situación (Castro y col., 2001).

Cabe añadir que los pacientes se están tornando más exigentes y reclaman mejores servicios (El Universal, 14/06/2008). Esta situación de por sí, bastante agobiante, puede agravarse ante la aparición de la crisis financiera mundial e incluso generar la disminución sustancial del número de clientes en las consultas privadas. Por su parte, la Organización Mundial de Salud (OMS) según lo plantea Chan (2009), en las crisis económicas la gente tiende a evitar la atención privada y hacer mayor uso de los servicios de carácter público. Esto lleva a deducir que las clínicas tendrán que cuidar mucho de la calidad del servicio ofrecido para evitar la pérdida de fidelidad por parte de los pacientes.
Una de las áreas más comprometidas en momentos de crisis socioeconómica según Chan (2009), es la pediatría, tomando en cuenta que son los niños quienes primero se verían afectados por el deterioro de la situación financiera y de la disponibilidad de alimentos. Entonces, en las consultas de pediatría, según la edad del niño, el mayor juez serán los padres o cuidadores que le llevan. Ante todo esto, se puede deducir que cuando un paciente $o$ cliente se siente insatisfecho por la atención recibida, se generan pérdidas tanto para él, como para la institución. El paciente muy posiblemente, no sentirá confianza, se sentirá frustrado y por lo tanto, no atenderá en su justa medida las instrucciones del médico, poniendo en riesgo la recuperación oportuna de su estado de salud e incluso, pueden causarle daños irreparables y también, es muy probable que nunca más regrese a este centro asistencial, hasta llegar a convertirse en un multiplicador de su rechazo a otros clientes, con la merma subsecuente de los ingresos y baja de la rentabilidad. De persistir esta situación de insatisfacción, el cliente definitivamente acudirá a otra institución de salud privada o pública y muy probablemente, cambiará de pediatra.

En ese contexto y tomando en cuenta que en el estado Falcón no hay información suficiente, sobre la satisfacción en el área de servicios pediátricos, se consideró pertinente determinar el nivel de satisfacción de los clientes de la Consulta Externa de Pediatría (CEP) en las Clínicas Privadas del Municipio Carirubana del Estado Falcón, a través de un estudio bajo un enfoque descriptivo y exploratorio, no experimental, así como la 
construcción de un sistema teórico en relación a las variables de investigación: Satisfacción del cliente y Servicios pediátricos en las clínicas privadas. El estudio se clasificó como un diseño de encuestas, porque se contactó directamente a los clientes de las consultas externas de pediatría, que funcionan en las clínicas privadas del municipio Carirubana.

La muestra fue indeterminada, no definida, de carácter intencional, opinática y estuvo constituida por las personas que solicitaron el servicio pediátrico en tres (3) de las clínicas que conforman la población, a saber: Policlínica de Especialidades, Clínica Falcón y Clínica La Familia (Tabla 1). Clínicas de fácil acceso para el investigador y sobre todo porque ofrecen mayor número de consultas externas de pediatría; lo que corresponde a un total de 24 CEP y 64 unidades informantes bajo estudio.

El instrumento constó de 44 ítems distribuidos en tres (03) partes: Datos del cliente (acompañante del paciente) que aportó el perfil sociodemográfico; registro de expectativas del cliente sobre la dinámica en las consultas externas de Pediatría y registro del rendimiento percibido; éste se aplicó posterior al momento en que el cliente fue atendido en la consulta.

\section{Caracterización del cliente de la consulta externa}

Tomando en cuenta a 64 clientes que solicitaron el servicio de consulta externa de pediatría en las clínicas del municipio Carirubana del estado Falcón, durante el período de 02 al 10 de julio de 2009 , se identificó y estableció el perfil sociodemográfico (Tabla 2).
Tabla 1. Categorización de la población y muestra

\begin{tabular}{lcc}
\hline \multicolumn{1}{c}{ Clínica } & CEP & Muestra \\
\hline Policlínica de & 8 & 12 \\
Especialidades & & \\
Policlínica Paraguaná & 6 & - \\
Clínica Falcón & 7 & 10 \\
Clínica La Familia & 9 & 42 \\
Centro Médico Cardón & 5 & - \\
Centro Médico & 3 & - \\
La Guadalupe & & \\
\hline
\end{tabular}

Fuente: Propia (2009).

Los resultados evidencian que en un $89,1 \%$ es la madre quien acude con el niño a la consulta, el 7,8\% corresponde al padre y apenas el $3,1 \%$ está representado por la abuela; cabe destacar que no se registró ninguna de las otras afiliaciones planteadas (abuelo, otro familiar y no familiar). Esto es una realidad, generalmente es la madre quien acude con el niño(a) a la consulta, incluso es quien le acompaña en los casos de hospitalización, tal como lo cita Galeano y col. (2006) refiriendo que en el $53 \%$ de los casos la madre era la responsable directa del niño internado. Es importante señalar que al momento de aplicar el instrumento y aun en los casos que ambos padres estaban presentes, fue la madre quien se ofreció a responder el cuestionario, en la mayoría de los casos.

En relación al género, un $92,2 \%$ de los encuestados pertenecen al sexo femenino y un $7,8 \%$ corresponde al género masculino. Sobre los grupos etáreos, $57,8 \%$ de los clientes (padres o representantes) están en edades comprendidas entre los 18 a 30 años. Al respecto, hay 
Tabla 2

\section{Caracterización del cliente}

de consulta externa de pediatría.

Clínicas privadas. Municipio

Carirubana. Estado Falcón. Perfil Sociodemográfico

\begin{tabular}{lll}
\hline Perfil Sociodemográfico & $f$ & $\%$
\end{tabular}

\section{Parentesco}

Madre

Padre

Otros (Abuela)

Género

Masculino

Femenino

Edad

$<18$

$18-30$

$31-43$

$>44$

Escolaridad

Analfabeta

Primaria

Secundaria

Superior

Frecuencia de uso de la consulta (al año)

Primera vez

De 2-5

$>6$

Motivo de consulta

Control niño sano

Enfermedad

Procedencia

$\begin{array}{lcc}\text { Rural } & 12 & 18,7 \\ \text { Urbana } & 52 & 81,3 \\ \text { Otra } & 0 & 0 \\ \text { ransporte } & & \\ \text { Propio } & 32 & 50 \\ \text { Público } & 32 & 50 \\ \text { Caminando } & 0 & 0 \\ \text { Otro } & 0 & 0\end{array}$

Referencia

Autoreferido

Otro

0 que destacar que Venezuela es un país, que se caracteriza por una población joven, donde el mayor porcentaje de padres corresponden a esas edades. En el grupo de 31 a 43 años, están un $29,1 \%$ y por último con el grupo de mayores de 44 años, se obtuvo un $3,1 \%$. Los clientes con estudios de educación superior correspondieron el 60,9\%; secundaria 32,8\% y primaria 6,3\%. Al respecto, Mezquita y col. (2008), refieren que la percepción según el nivel de escolaridad (sólo estudios primarios vs secundarios) no fue diferente, sin embargo en el presente estudio, pudiera haber influido en la percepción, la formación profesional, lo cual motiva a futuras investigaciones.

En el mismo orden de ideas, Galeano y col. (2006) plantean que en un estudio realizado por ellos, en un hospital público, el 76\% presentaba niveles educativos de riesgo por no haber concluido la escolarización primaria o por alcanzar sólo el primer o segundo año de la secundaria, caso contrario se evidencia en el presente estudio, donde 21 clientes, que representan un $32,8 \%$, corresponden a clientes con niveles educativos de riesgo. Cabe resaltar que al comparar estos resultados debe tomarse en consideración que son dos sectores distintos, uno público y el otro privado, lo cual puede afectar la variable en estudio "satisfacción del cliente".

La frecuencia de uso de la consulta fue en un $51,6 \%$ de 2 a 5 veces al año, los que acudieron por primera vez 32,8 $\%$, los que acudieron más de 6 veces $15,6 \%$. Al respecto Reeve (1994) y Thompson (2005), refieren que las expectativas se crean y cambian, entre otros aspectos, por experiencia directa;

Fuente: Propia (2009). 
vale destacar, que con estos resultados se pudiera vislumbrar rasgos de fidelidad en el paciente.

El motivo de consulta para realizar el control de niño sano fue de $57,8 \%$ y $42,2 \%$ por enfermedad. En tal sentido, Mezquita y col. (2008), en estudio realizado en hospitales públicos de la Asunción y Gran Asunción de Paraguay, detectó que el $58,7 \%$ de los niños acudió por enfermedad. En el caso objeto de estudio de esta investigación el hecho de predominar el control de niño sano como motivo de consulta, aunado a lo referido en el párrafo anterior, pudiera reforzar el concepto de rasgos de fidelidad con la consulta. Esto lleva por cierto, a la reflexión sobre la existencia de una cultura de prevención de los clientes que acuden con sus niños a las clínicas privadas, o bien que prefieren para control de niño sano una institución privada.

Con respecto a la procedencia, los clientes que provienen del medio urbano equivalen al $81,3 \%$ y solo un $18,7 \%$, provienen del medio rural. Esto puede obedecer a que los consultorios objeto de estudio están ubicadas en clínicas urbanas. En cuanto al transporte que usaron para trasladarse al centro asistencial, un 50\% de los entrevistados manifestaron usar el transporte público para acudir al centro asistencial; igual proporción manifestó emplear vehículo propio.

En relación a la fuente de referencia, los 64 clientes que conforman la muestra (100\%), acudieron por decisión propia. No fueron referidos por empresa laboral o por disfrutar de una póliza de seguro de hospitalización, cirugía o maternidad. Diferente ocurre con lo reportado en el estudio de Silva (2005) al investigar sobre la calidad de los servicios privados de salud del Municipio Maracaibo, estado Zulia. En dicho estudio, Silva detectó que los motivos para elegir un centro asistencial están dados principalmente por la fuente de remisión (remisión del seguro y/o empresas) y las experiencias previas; la familia y los grupos de referencia, se apreciaron en menor escala. De igual manera, la remisión medica, el galeno tratante y la ubicación geográfica tienen muy poca importancia.

Sin embargo, ante lo mencionado con respecto al medio de transporte y la fuente de referencia, es pertinente reflejar que el cliente acude al servicio privado, tal como lo menciona Tapia (2007), atraído por diferentes factores. En esta investigación ninguno llegó caminando, dejando claro que no viven dentro de la llamada zona base, por lo cual no estaban en la búsqueda de un factor de conveniencia y servicio rápido. Por lo cual pudiera afirmarse que los clientes objeto de estudio, buscan un factor de buen servicio y cartera surtida de servicios, o bien, aquellos que provienen del medio rural, buscan un factor de productos únicos y buen precio. En la Tabla 3 se presentan los criterios referidos para acudir a la consulta, entre los que destacan acudir por recomendación de familiares o amigos, experiencias previas positivas y por ubicación geográfica del centro asistencial.

En el mismo orden de ideas, algunos clientes expresaron haber manejado más de un criterio para seleccionar la consulta. Es así como un 15,6\% decidieron tomando en cuenta la ubicación geográfica y experiencias previas positivas; mientras que el 6,3\% consideraron en conjunto, la ubicación geográfica y reco- 


\section{Tabla 3}

Criterios de selección de la consulta

\begin{tabular}{lcc}
\hline \multicolumn{1}{c}{ Criterios } & $\mathrm{f}$ & $\%$ \\
\hline La ubicación geográfica & 13 & 20,3 \\
$\begin{array}{l}\text { La recomendación de } \\
\text { familiares o amigos }\end{array}$ & 17 & 26,6 \\
$\begin{array}{l}\text { Mis experiencias previas } \\
\text { positivas }\end{array}$ & 14 & 21,9 \\
Otras & 20 & 31,2 \\
\hline
\end{tabular}

Fuente: Propia (2009).

mendación de familiares o amigos; y un $9,2 \%$ por recomendación de familiares o amigos y por experiencias previas positivas. Finalmente, se puede inferir que la decisión del cliente de acudir a la clínica privada se debe a la situación actual de las instituciones públicas de salud en Venezuela, debido al escaso crecimiento de la salud pública (EI Nacional, 16/9/2008; El Universal, 28/1/2008).

En base a los aspectos descritos con respecto a la estimación del perfil sociodemográfico de los clientes objeto de estudio, cabe destacar que aunque Silva (2005) atribuye una amplia diversificación de criterios para identificar las características de los clientes, mencionando incluso que estos pueden tener cualquier edad, sexo y procedencia. En síntesis, se logró establecer el perfil sociodemográfico de los clientes de consulta externa de pediatría de las clínicas del municipio Carirubana del estado Falcón, el cual quedó caracterizado así: madre, obviamente género femenino, 18 a 30 años de edad, con estudios de educación superior, que asiste a la consulta más de dos veces al año, a control de niño sano, procede del medio urbano, se moviliza en vehículo propio o público hasta la clínica y no es referida por empresa laboral o de seguros, eligiendo a la consulta principalmente en base a la recomendación de familiares o amigos.

\section{Expectativas y Rendimiento percibido por los clientes}

\subsection{Expectativas}

Tomando en cuenta el significado de la expectativa de una persona cuando solicita un servicio, Reeve (1994) la cataloga como la evaluación subjetiva de la probabilidad de alcanzar una meta concreta, o también Thompson (2005), le considera como la esperanza que los clientes tienen por conseguir algo. En referencia a ello, se presentan a continuación las expectativas referidas por los clientes o unidades de información bajo estudio.

En el mismo orden de ideas, una vez establecido el perfil sociodemográfico y los criterios que sustentaron la selección de la consulta por parte del cliente, es importante señalar las expectativas que ellos tienen sobre la consulta, lo cual se evidencia en la Tabla 4.

Del total de clientes, un elevado porcentaje $96,9 \%$, afirmaron esperar recibir atención amable del personal; el 100\% confía en realizar un trámite sencillo para la asignación de la cita; 96,9\% esperaban ser atendidos por un personal de secretaría competente. De igual manera, los resultados del estudio evidencian que el $100 \%$ de los encuestados mantienen la expectativa de ser atendidos por un pediatra competente; en el mismo orden de ideas, elementos como la confidenciali- 


\section{Expectativas sobre la consulta externa de pediatría}

\begin{tabular}{lcccc}
\hline \multirow{2}{*}{ Aspectos del servicio } & \multicolumn{2}{c}{ Sí } & \multicolumn{2}{c}{ No } \\
\cline { 2 - 5 } & $\mathrm{f}$ & $\%$ & $\mathrm{f}$ & $\%$ \\
\hline Atención amable del personal & 62 & 96,9 & 2 & 3,1 \\
Tramite sencillo para la asignación de la cita & 64 & 100 & 0 & 0 \\
Personal de secretaria competente & 62 & 96,9 & 2 & 3,1 \\
Pediatra competente & 64 & 100 & 0 & 0 \\
Confidencialidad por parte del personal & 62 & 96,9 & 2 & 3,1 \\
Tiempo de espera corto & 60 & 93,8 & 4 & 6,3 \\
Tiempo de duración del acto médico suficiente & 64 & 100 & 0 & 0 \\
Ambiente confortable & 62 & 96,9 & 2 & 3,1 \\
Adecuada apariencia física de las instalaciones & 61 & 95,3 & 3 & 4,7 \\
Equipos médicos en buen estado & 63 & 98,4 & 1 & 1,6 \\
Tecnología moderna & 62 & 96,9 & 2 & 3,1 \\
Precio de la consulta accesible & 64 & 100 & 0 & 0 \\
\hline
\end{tabular}

Fuente: Propia (2009).

dad por parte del personal fue esperado por los clientes en un $96,9 \%$.

Con respecto al tiempo de espera corto fue deseado por un alto porcentaje $93,8 \%$. El $100 \%$ esperaban que la duración del acto médico fuese suficiente. Un ambiente confortable era aspirado por el $96,9 \%$ de los encuestados y sólo un $3,1 \%$ estuvieron en desacuerdo con las actuales condiciones. Con respecto a la adecuada apariencia física de las instalaciones, un $95,3 \%$ lo esperaban. El buen estado de los equipos médicos, fue un requerimiento del $98,4 \%$ de los encuestados. De igual manera, el hecho de que la clínica contara con tecnología moderna era una condición deseable por el $96,9 \%$. Otro indicador como el precio accesible de la consulta era esperado por el $100 \%$ de la muestra consultada.

Una vez identificadas las expectativas de los clientes sobre el servicio a ser prestado en la consulta externa de pediatría de las clínicas encuestadas, y en concordancia con lo destacado por Schiffman y Lazar (2001), quienes manifiestan que la gente tiende a percibir los productos y atributos de los productos y servicios, de acuerdo con sus propias expectativas. En relación con lo expresado, se presentan a continuación los resultados obtenidos en cuanto al rendimiento percibido, con respecto a la estancia y condiciones físicas de la clínica (Tabla 5), atención pediátrica (Tabla 6) y satisfacción global (Tabla 7).

\subsection{Rendimiento percibido por los clientes}

El rendimiento percibido, como lo refiere Thompson (2005) se determina desde el punto de vista del cliente, no de la empresa; se basa en los resultados 


\section{Tabla 5}

Estancia y Condiciones Físicas: Rendimiento percibido

\begin{tabular}{|c|c|c|c|c|c|c|}
\hline \multirow[t]{2}{*}{ Aspectos } & \multicolumn{2}{|c|}{ Nada satisfecho } & \multicolumn{2}{|c|}{$\begin{array}{l}\text { Más o menos } \\
\text { satisfecho }\end{array}$} & \multicolumn{2}{|c|}{ Satisfecho } \\
\hline & $f$ & $\%$ & $f$ & $\%$ & $f$ & $\%$ \\
\hline Primera impresión & 0 & 0 & 7 & 10,9 & 57 & 89,9 \\
\hline Apariencia física del personal & 0 & 0 & 3 & 4,7 & 61 & 95,3 \\
\hline Relación interpersonal & 0 & 0 & 6 & 9,4 & 58 & 90,6 \\
\hline $\begin{array}{l}\text { Atención y ayuda del personal } \\
\text { administrativo }\end{array}$ & 0 & 0 & 11 & 17,2 & 53 & 82,8 \\
\hline Apariencia de las instalaciones & 1 & 1,6 & 5 & 7,8 & 58 & 90,6 \\
\hline $\begin{array}{l}\text { Estado de equipos médicos y } \\
\text { tecnológicos }\end{array}$ & 1 & 1,6 & 3 & 4,7 & 60 & 93,8 \\
\hline Tiempo de espera & 1 & 1,6 & 10 & 15,6 & 53 & 82,8 \\
\hline Temperatura de la sala de espera & 2 & 3,1 & 4 & 6,3 & 58 & 90,6 \\
\hline Sala de espera libre de ruidos & 2 & 3,1 & 11 & 17,2 & 51 & 79,7 \\
\hline $\begin{array}{l}\text { Estado de limpieza de sala de } \\
\text { espera }\end{array}$ & 0 & 0 & 2 & 3,1 & 62 & 96,9 \\
\hline Estado de limpieza del consultorio & 0 & 0 & 1 & 1,6 & 63 & 98,4 \\
\hline Mobiliario de sala de espera & 1 & 1,6 & 7 & 10,9 & 56 & 87,5 \\
\hline
\end{tabular}

Fuente: Propia (2009).

\section{Tabla 6}

Atención pediátrica: Rendimiento percibido

\begin{tabular}{lcccccc}
\hline \multicolumn{1}{c}{ Aspectos } & \multicolumn{2}{c}{ Nada satisfecho } & \multicolumn{2}{c}{$\begin{array}{c}\text { Más o menos } \\
\text { satisfecho }\end{array}$} & \multicolumn{2}{c}{ Satisfecho } \\
\cline { 2 - 7 } & $\mathrm{f}$ & $\%$ & $\mathrm{f}$ & $\%$ & $\mathrm{f}$ & $\%$ \\
\hline Tiempo de atención en la consulta & 0 & 0 & 1 & 1,6 & 63 & 98,4 \\
Al preguntarle sobre las vacunas & 0 & 0 & 1 & 1,6 & 63 & 98,4 \\
Al preguntarle sobre la evolución & 0 & 0 & 1 & 1,6 & 63 & 98,4 \\
psicomotora & & & & & & \\
Examen físico & 0 & 0 & 0 & 0 & 64 & 100 \\
Explicación sobre el diagnóstico & 0 & 0 & 1 & 1,6 & 63 & 98,4 \\
Explicación sobre el tratamiento & 0 & 0 & 1 & 1,6 & 63 & 98,4 \\
\hline
\end{tabular}

Fuente: Propia (2009). 


\section{Tabla 7
Satisfacción global (Fidelización)}

\begin{tabular}{lcccc}
\hline \multirow{2}{*}{ Valor } & \multicolumn{2}{c}{ Sí } & \multicolumn{2}{c}{ No } \\
\cline { 2 - 5 } & $\mathrm{f}$ & $\%$ & $\mathrm{f}$ & $\%$ \\
\hline Elegiría de nuevo venir a esta consulta & 64 & 100 & 0 & 0 \\
Se cambiaría de consulta & 1 & 1,6 & 63 & 98,4 \\
Se cambiaría de clínica & 8 & 12,5 & 56 & 87,5 \\
Recomendaría esta consulta & 64 & 100 & 0 & 0 \\
\hline
\end{tabular}

Fuente: Propia (2009).

que el cliente obtiene con el producto o servicio; está basado en las percepciones del cliente, no necesariamente en la realidad; sufre el impacto de las opiniones de otras personas que influyen en el cliente y depende del estado de ánimo del cliente y de sus razonamientos.

Los resultados del estudio expresados en la Tabla 5, en cuanto a los aspectos del servicio que produjeron satisfacción en todos los clientes, relacionados con la estancia y condiciones físicas de las instalaciones hospitalarias, obtuvieron porcentajes elevados. Concuerdan estos resultados con la apreciación de Buitrago (2007) quien lo considera como unos de los cuatro factores que dan satisfacción al cliente, junto con la conveniencia, respeto y cuidado $y$, efectividad y continuidad.

Cabe destacar que aunque la mayoría está satisfecho con la relación y apoyo del personal, es pertinente recordar lo referido por Mezquita y col. (2008) al afirmar que el buen trato personal es un factor preponderante en la calidad de la atención (personalizada y humanizada), así como las explicaciones brindadas sobre el problema del paciente. Por su parte, es relevante destacar que según los resultados presentados por Silva (2005) el servicio de apoyo de administración, no genera mucha satisfacción en los clientes que hacen uso de los servicios medico-asistenciales y aspectos como la apariencia de las instalaciones y el estado de los equipos médicos son importantes como generadores de satisfacción en el cliente, tal como lo expresa Jelenkovic (2007); lo cual concuerda con los resultados obtenidos en la presente investigación. De igual manera coinciden los resultados de Mesquita y col. (2008) y Jelenkovic (2007), en relación a que los pacientes le dieron importancia a las comodidades en la sala de espera, pero agregaron que un área de juego para los niños, representaba un punto de referencia para generar satisfacción.

Es importante señalar que en el presente estudio, de los aspectos relacionados con la atención pediátrica (Tabla 6), promediando todos los indicadores, un $98 \%$ consideran estar satisfechos con el tiempo de atención en la consulta, y aquellos indicadores relacionados con el examen físico, evaluación psicomotora, vacunas; así como la explicación en cuanto al diagnóstico y tratamiento.

En resumen, se determinó que los clientes de los consultorios pediátricos de 
Clínicas privadas del Municipio Carirubana del estado Falcón están satisfechos con el servicio recibido.

La medición que se realizó sobre la satisfacción global (Tabla 7) que orienta hacia la fidelización del cliente, determinó que en los consultorios pediátricos de clínicas privadas del Municipio Carirubana, son acreedores de un estado de satisfacción global, que le induce a dar un valor positivo a los planteamientos presentados, lo cual seguramente le proporcionará a los centros asistenciales consultados grandes beneficios, como lo es la lealtad, la difusión gratuita de sus experiencias positivas con el servicio y dejará de lado a la competencia. Cabe destacar la apreciación de Barahona (2006) quien cataloga la fidelidad del cliente como una actitud positiva que supone la unión de la satisfacción del cliente, formada por elementos racionales y afectivos, con una acción de consumo estable y duradero.

\section{Conclusiones}

Como parte de los resultados del estudio, se obtuvo que los clientes de la consulta externa de Pediatría son predominantemente, madres jóvenes, instruidas, que aplican criterios de prevención para promover la salud de sus hijos, que supera la distancia existente para acudir al pediatra y clínica que seleccionó, y que además, recibe y escucha orientación de personas con experiencias previas positivas.

De igual manera, los clientes acuden a la consulta con una actitud positiva al poder seleccionar la clínica y el pediatra, motivación inducida por los resultados de sus experiencias previas y por la influencia de familiares y amigos. En concordancia con la teoría de las necesidades humanas de Abraham Maslow, los clientes solicitan atención médica por necesitar primordialmente, seguridad, afiliación y afecto, y también, estima. Es decir, acuden en la idea de conocer el estado de salud o de recuperarla, así como, buscando encontrar signos de estima al solicitar el servicio. En tal sentido, el condicionamiento previo de los clientes favorecido por la experiencia directa positiva y reforzado por la experiencia y persuasión verbal de familiares y amigos, aunado a las características de su perfil mencionadas anteriormente, favorecieron la formación de expectativas de alto nivel.

Las expectativas de los clientes con casi todos los aspectos presentados, principalmente con el trámite sencillo para la asignación de la cita, pediatra competente, tiempo de duración del acto médico suficiente y precio de la consulta accesible, muestran a un cliente con elevado nivel de exigencia, que está seguro de recibir un servicio de calidad, lo cual se hace mayor tomando en cuenta su perfil sociodemográfico. Además, muy convencido que tendría que esperar cierto tiempo para ser atendido y que se encontraría con instalaciones de la clínica no del todo satisfactorias.

El rendimiento que los clientes percibieron del servicio aportado por la clínica, están influenciados por sus altas expectativas y su perfil, sin descartar, por supuesto, el estado de ánimo que el cliente pudo haber tenido en ese momento. Así lo demuestran, la satisfacción, principalmente con la primera impresión recibida, la apariencia física del personal, la relación interpersonal desarrollada, la aten- 
ción y ayuda del personal administrativo recibida, y además, la satisfacción por el examen físico que le fue realizado al paciente. A esto se suma, las bajas expectativas que tenían sobre la apariencia y estado de limpieza de las instalaciones, el estado de equipos médicos y tecnológicos, el tiempo de espera, el mobiliario y sobre todo las condiciones de confort en las salas de espera en cuanto temperatura ambiental y nivel de ruidos, aspectos con los cuales algunos no mostraron satisfacción.

El nivel de satisfacción fue suficiente como para despertar fidelización en los clientes, puesto que declararon elegirían volver y le recomendarían a un familiar o conocido acudir a esa consulta, dejando excluida cualquier oferta que pudiese surgir de otras clínicas u otros pediatras. En esto también influye la relación permanente que sostienen con el pediatra y con la clínica. Tomando en cuenta que las personas deciden cual es su grado de satisfacción al comparar de manera consciente sus necesidades y circunstancias, en este caso, sus necesidades y expectativas se conjugaron con las circunstancias, es decir con el rendimiento percibido. Todo ello refuerza su motivación para acudir a la consulta, y además, son clientes satisfechos por habérseles cumplido sus expectativas, aunque no de manera absoluta, siendo muy probable que acudan nuevamente con el mismo pediatra y en la misma clínica.

En síntesis, el rendimiento percibido por los clientes de las consultas externas de pediatría en las clínicas privadas del municipio Carirubana del estado Falcón es satisfactorio, pero no absoluta- mente satisfactorio, por lo cual en las consultas externas de pediatría y por supuesto, en las clínicas en donde están ubicadas, deben implementarse estrategias para lograr la satisfacción absoluta del cliente y garantizar su fidelidad con el servicio.

\section{Referencias bibliográficas}

Barahona, Alfonso (2006). Atención al cliente en el siglo XXI. http://www.monografias.com/trabajos66/atencion-clientesigloxxi/atencioncliente-siglo-xxi2. shtml. Consultado: 14 de julio de 2009.

Buitrago González, Misael (2007). Satisfacción de los clientes con los servicios hospitalarios en el Área de Mayagüez, Puerto Rico. Tesis en Maestro en Administración de Empresas. Universidad de Puerto Rico Recinto Universitario de Mayagüez.

Castro, Mary; Morillo, Jorge; Iglesias, Josneyda (2001). Satisfacción de usuarios en las consultas preventivas del Centro Asistencial "José María Espinoza”. Coro, estado Falcón. Acta Científica Venezolana 52(Supl. 3): 234.

Chan, Margaret (2009). La crisis financiera y la salud mundial. OMS. Disponible en: http://www.who.int/dg/speeches/ 2009/financial_crisis_20090119/es /index. html. Consultado: 03 de febrero de 2009.

El Nacional (16/9/2008). Las clínicas trabajan a máxima capacidad. Noticias y Titulares de Venezuela. Disponible en: http://www.guia.com.ve/noticias/?id= 28802. Consultado: 05 de octubre de2008.

EI Universal (14/6/2008). Usan listas de espera en las clínicas privadas debido a su saturación. Noticias y Titulares de Ve- 
nezuela. Disponible en: http://www. guia. com.ve/noticias/?id=24297.

Consultado: 05 de agosto de 2009.

El Universal (28/1/2008). Clínicas están obligadas a crecer. Noticias y Titulares de Venezuela. Disponible en: http:/ /www.guia.com.ve/noticias/?id= 17637. Consultado: 05 de marzo de 2009.

Galeano, Humberto; Furlán, Claudia; Auchter, Mónica; Balbuena, Mirtha; Zacarías, Gladis; Zacarías, Lidia (2006). Nivel de satisfacción con la atención de enfermería en un hospital pediátrico. Hospital Pediátrico Juan Pablo II, Cátedra Enfermería Maternoinfantil, UNNE, Argentina. Disponible en: http://www.unne.edu.ar/Web/cyt/cyt2 006/03-Medicas/2006-M-134.pdf.

Consultado: 12 de febrero de 2009.

Huete, Luis (2001). Servicios y Beneficios. Bilbao: Deusto.

Horovitz, J. y Jurgens, M. (1999). La Satisfacción Total del Cliente. Madrid: McGraw-Hill.

Jelenkovic, Zlata (2007). El enfoque de satisfacción del cliente en los centros de atención médica privada (Caso Consultas externas de Medicina Interna en las clínicas privadas de Paraguaná). Punto Fijo, Estado Falcón. Tesis en Gerencia de Empresas Mención Mercadeo. No publicado. Universidad del Zulia. Núcleo Punto Fijo.

Kotler, Philip (1993). Dirección de Mercadotecnia. Análisis, Planeación, Implementación y Control. Prentice Hall-México.

Labrador, Hender (2006). La Satisfacción del Cliente. CIDEC. Disponible en: http: //www.henderlabrador.galeon.com. Consultado: 08 de marzo de 2009.

Mesquita, M; Pavlicich, V; Benitez, S (2008). Percepción de la calidad de atención y accesibilidad de los consultorios pe- diátricos públicos en Asunción y Gran Asunción. Paraguay. Pediatría (Asunción), 35(1).

Oliver, R. (1980). A cognitive model of the antecedents and consequences of satisfaction decision. Journal of Marketing Research 17: 460-469.

Peralta Montesinos, Jeniffer (2006). Rol de Expectativas en el juicio de Satisfacción y Calidad Percibida del Servicio. Universidad de Tarapacá. Arica, Chile. Revista de Filosofía y Psicología. 1(14): pp. 195-214. Disponible en: http://www.dialnet.unirioja.es/servlet/ fichero_articulo?codigo $=2252855 \&$ orden=77449. Consultado: 20 de abril de 2009.

Reeve, Johnmarsahll (1994). Motivación y emoción. McGraw-Hill. Madrid.

Schiffman, Leon; Lazar, Leslie (2001). Comportamiento del Consumidor. $7^{\mathrm{a}} \mathrm{ed}$. México: Prentice Hall.

Silva, Yelitza (2005) Calidad de los servicios privados de salud. Revista Ciencias Sociales LUZ. XI (001). pp. 167-177.

Tapia, Jaime (2007). Gestión de servicios: La Consulta Pediátrica. Serie de Reportajes Herramientas de Gestión para el pediatra actual Sociedad Chilena de Pediatría No3, año 6. 2007. Disponible en http://www.sochipe.cl/nueva/html/ estetoscopio/3-6/6_3_consulta.htm. Consultado: 08 de abril de 2009.

Thompson, Iván (2005). La Satisfacción del Cliente. Promonegocios.net. Disponible en http://www.promonegocios.net /mercadotecnia/satisfaccion-cliente. htm. Consultado: 20 de mayo de 2009.

World Health Organization (2006). Concepts, methods and debates [serie en Internet]. Disponible en: http://www.who. int/health-systems-performance/concepts.htm. Consultado: 02 de marzo de 2009 . 